\title{
The Role of Gold Clusters in Semiconductor Microstructure Fabrication
}

\author{
Masataka Shirai, Keichi Haraguchi, Kenji Hiruma, Toshio Katsuyama \\ Central Research Laboratory, Hitachi Ltd, Kokubunji, Tokyo 185-8601, Japan
}

Received: 1 June 1999

Semiconductor wire-like microstructures with nanometer-scale dimensions have been grown by a selforganized technique. This fabrication is based on organometallic vapour-phase epitaxy (OMVPE). Gold is a key material for growing such nanometer-cylinders (called whiskers). Artificially controlling the gold deposition and the subsequent self-organized growth process resulted in successful control of diameter, length, and growth position of whiskers. Low-damage quantum-wire structures were fabricated which showed quantum confinement effects for electrons in quasi one-dimensional-structures. As an application of these structures, GaAs whiskers with p-n junctions were grown and used in quantum-wire light emitters.

Current fabrication techniques for semiconductor fine structures are also useful for fabricating onedimensional structures that show quantum size effects in electrical and optical properties. Various techniques have been used to make such wire structures $(1,2)$. Ion beam lithography (3), ion etching (4), molecular beam epitaxy (MBE) (2), and organometallic vapour-phase epitaxy (OMVPE) (5) are especially suitable and reliable techniques for this purpose. However, wires formed by ion beams and ion etching are subject to damage or contamination at the crystal surface.

We previously fabricated a semiconductor wire structure consisting of $\mathrm{GaAs}$ and InAs whiskers grown by OMVPE $(6,7)$ with gold as a growth catalyst. Those whiskers can provide damage-free quantum-size wire crystals because they allow direct formation of structures as small as $20 \mathrm{~nm}$ without using etching techniques.

A growth procedure for silicon whiskers in the vapour phase using gold was first reported by Wagner and Ellis (8), and they proposed a vapour-liquid-solid (VLS) mechanism to explain the whisker shape. Givargizov (9) reported growth kinetics of $\mathrm{Si}, \mathrm{Ge}$, and $\mathrm{SiC}$ whiskers grown by vapour phase epitaxy using gold as a catalyst. However, a suitable method for controlling the size, shape, growth direction or growth position of whiskers has not previously been reported.

We fabricated GaAs and InAs whiskers by controlling their shapes, directions and positions.
We examined their structures by scanning electron microscopy (SEM) and transmission electron microscopy (TEM), and measured the optical properties of LEDs fabricated by using those whiskers.

\section{WHISKER GROWTH BY OMVPE}

\section{Growth Process}

SEM photographs of typical GaAs whiskers formed on a $\mathrm{GaAs}(111) \mathrm{As}$ (GaAs(111)B) substrate surface at $420^{\circ} \mathrm{C}$ is shown in Figure 1. The direction of whisker growth is parallel to the (111)As direction.

The experimental procedure for growing the $\mathrm{GaAs}$ whiskers is illustrated in Figure 2. First, gold was deposited on a GaAs(111)As substrate by conventional vacuum evaporation. The average thickness of the deposited gold varied between 0.1 and $10 \mathrm{~nm}$. After gold deposition, the substrate was exposed to air before being placed in an OMVPE growth chamber, which was heated at $500^{\circ} \mathrm{C}$ for about $10 \mathrm{~min}$ in an OMVPE atmosphere. Wires were then grown along the (111)As direction by supplying the source gases trimethylgallium (TMG) and arsine $\left(\mathrm{AsH}_{3} ; 10 \%\right.$ in $\mathrm{H}_{2}$ ).

The probable mechanism of whisker growth is explained as follows. The gold deposited on the substrate surface forms islands when the average gold thickness is ca $0.1 \mathrm{~nm}$ (Figure 2(a)). During the 


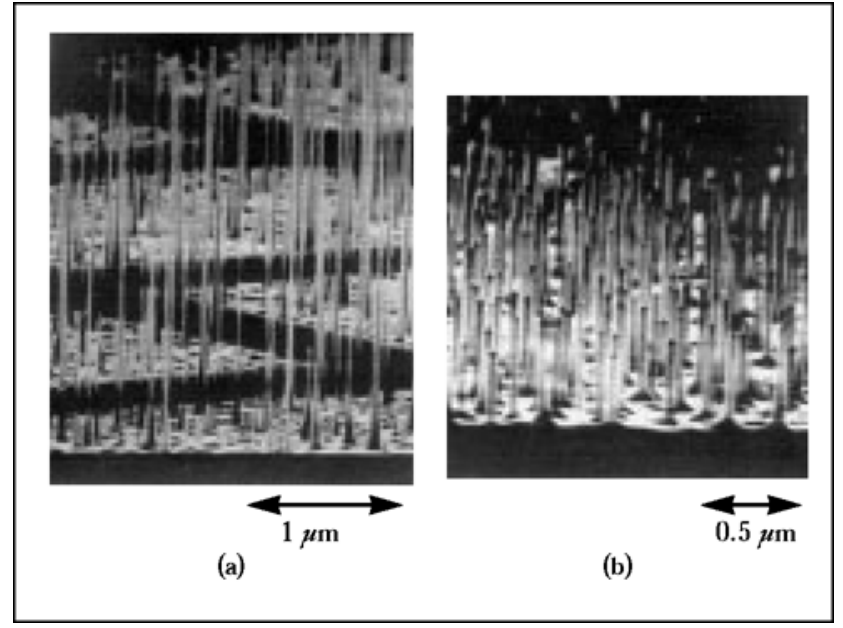

Figure 1 SEM photographs of typical GaAs whiskers formed on a GaAs(III)As (GaAsIII)B) substrate surface at $420^{\circ} \mathrm{C}$

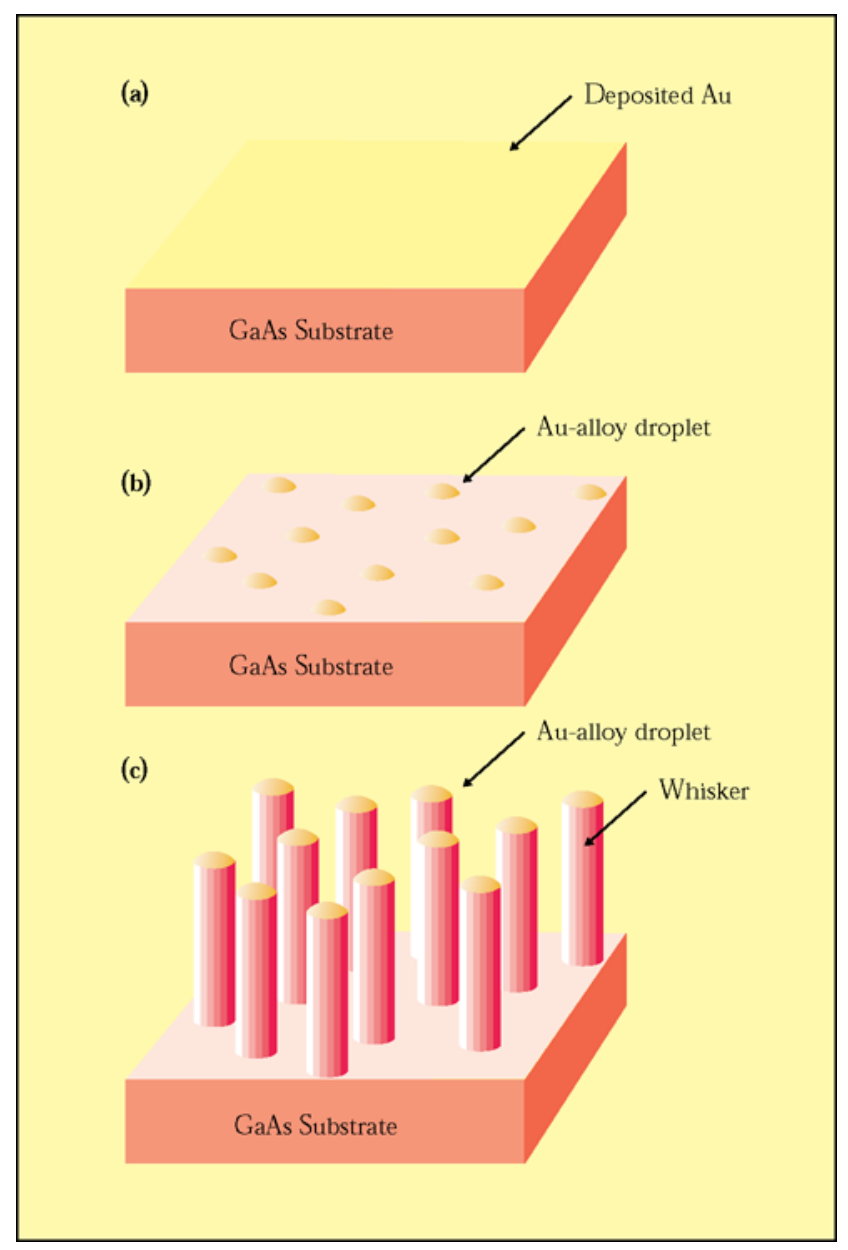

Figure 2 Representation of experimental procedure for growing GaAs whiskers. heating in an $\mathrm{AsH}_{3} / \mathrm{H}_{2}$ atmosphere, Au-Ga-As alloy droplets are formed by a reaction between the gold and the GaAs substrate (Figure 2(b)). When the thickness of the deposited gold is larger than $0.1 \mathrm{~nm}$, the same reaction probably proceeds during the heating. The alloy droplets were as small as $10 \mathrm{~nm}$ when the average thickness of deposited gold was $0.1 \mathrm{~nm}$. During the heating in OMVPE, the amount of source molecules absorbed into the gold alloy increases with increasing supply of $T M G$ and $\mathrm{AsH}_{3}$. This increased supply causes a supersaturated condition for $\mathrm{Ga}$ and $\mathrm{As}$ in the alloy. Thus, GaAs deposits on the $\mathrm{GaAs}(111)$ As crystal face, and wire-shaped whiskers are grown as shown in Figure 2(c).

Our experimental results show that the whiskers grown at about $400^{\circ} \mathrm{C}$ are cylindrical in shape and their width is almost equal to the diameter of the AuGa-As alloy at the tip. We therefore believe that the growth process is governed by the VLS growth mechanism proposed by Wagner and Ellis. As shown in Figure 3, actual alloy droplets can be seen on the top of the wire crystals. The grown whiskers are cylindrical and about $15 \mathrm{~nm}$ in diameter.

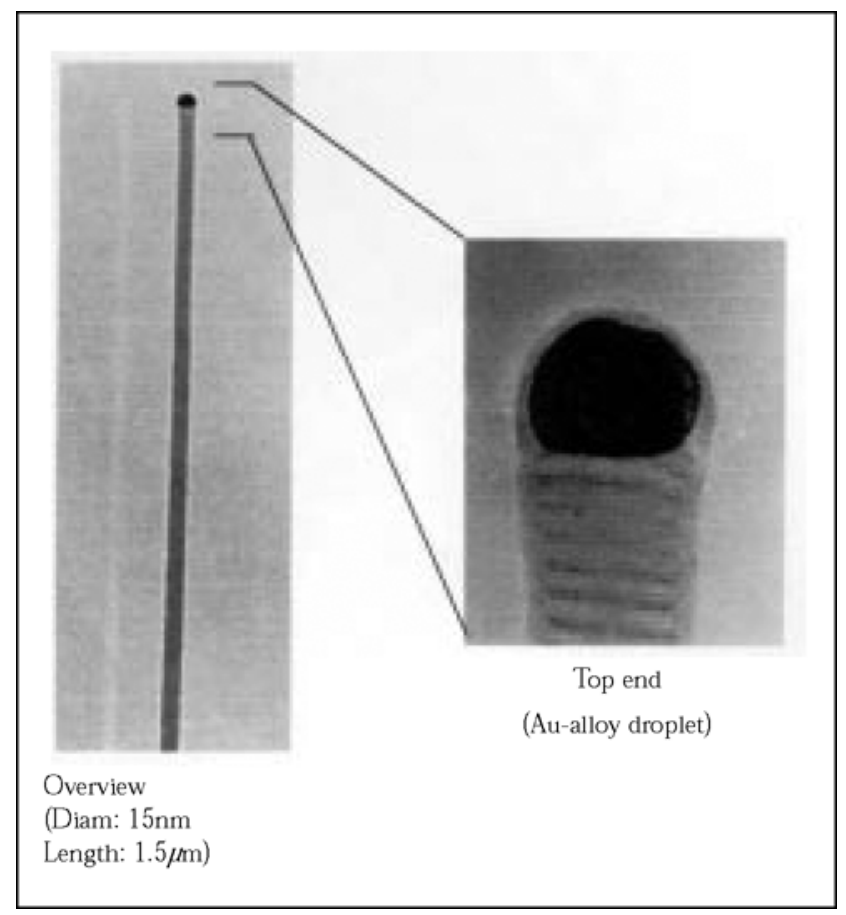

Figure 3 GaAs whisker growth. 


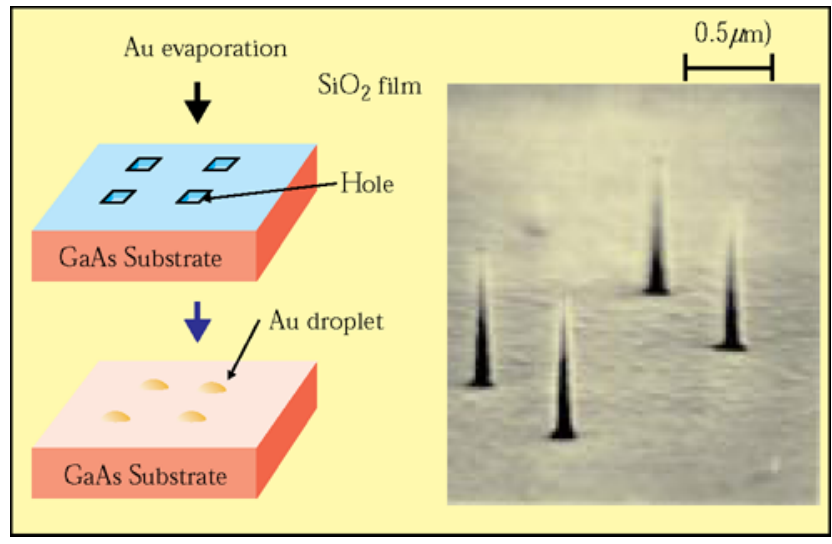

Figure 4 SEM image of site-controlled GaAs wine crystals grown on GaAs(III)B substrate.

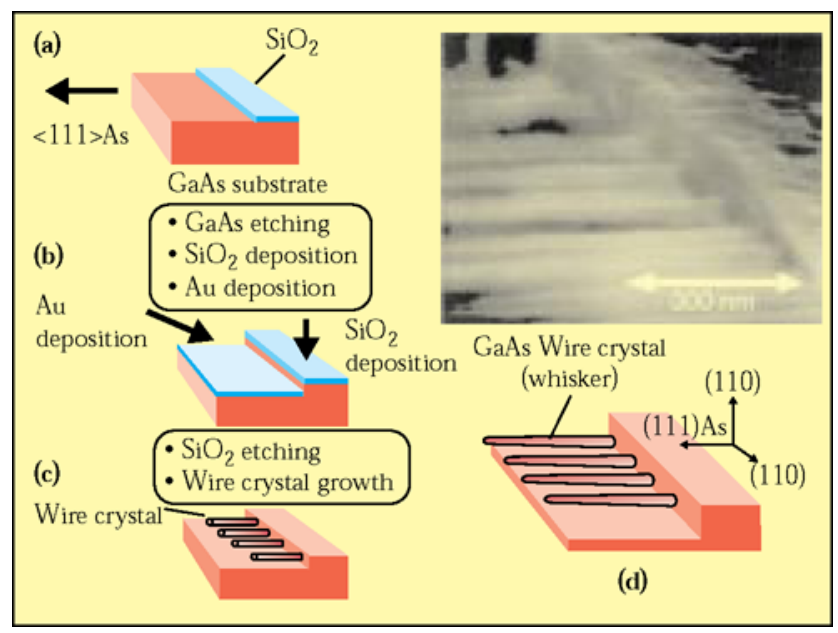

Figure 5 Growth of lateral whiskers on GaAs.

\section{Growth Control}

We first artificially controlled the growth position of the wire crystal (10). Such control is essential for various device applications for whiskers. The growth procedure for site-controlled whiskers is shown schematically in the inset of Figure 4. We controlled the location of whiskers by using a silica window mask formed by electron-beam lithography. After removing the patterned resist layer with a $1 \mathrm{~nm}$-thick gold thin film, the gold film remained only inside the silica windows. The annealing process formed a single alloy droplet inside each window. After removing the silica mask, the sample was placed in an MOVPE chamber to grow the whiskers. Figure 4 shows an SEM image of site-controlled GaAs wire crystals grown on a $\mathrm{GaAs}(111) \mathrm{B}$ substrate. Wire crystals with diameters of about $80 \mathrm{~nm}$ are placed exactly at the four corners of a $2 \times 2 \mu \mathrm{m}$ square.
Next, we fabricated the lateral whiskers as described in Reference 11. To grow the whiskers laterally, gold-alloy droplets must be formed on the side wall of a ridge formed on the substrate. This was achieved by selecting a GaAs substrate whose [111]As direction was normal to the side edge of the $\mathrm{SiO}_{2}$ pattern (Figure 5(a)). GaAs (110) and (211) As substrates were used. The $\mathrm{SiO}_{2}$ masking pattern was formed by conventional photolithography. Then the substrate was partially etched away and a step (about 5 $\mu \mathrm{m}$ high) was formed. $\mathrm{SiO}_{2}$ was deposited again on the entire substrate surface except for the side wall of the $\mathrm{GaAs}$ step. A small amount of gold (less than one monolayer) was evaporated onto the substrate, which was then heat-treated at $500^{\circ} \mathrm{C}$ (Figure 5(b)). Wire crystals were grown by MOVPE after the silica mask had been removed (Figure 5(c)). The wire crystals grown were about 30-60 $\mathrm{nm}$ in diameter and about 2 $\mu \mathrm{m}$ long. Figure 5(d) illustrates an SEM image and a schematic configuration of the GaAs whiskers.

When growth time is sufficiently long, bridge-type whisker arrays can be fabricated by using a technique similar to the one described above (11). Figure 6 shows the bridge-type GaAs whisker arrays we fabricated. The substrate used for this experiment had a shallow $1 \mu \mathrm{m}$ wide ditch. One side wall of the ditch has a (111)As face, and the other a (111) $\mathrm{Ga}$ face.

\section{WHISKERS WITH P-N JUNCTIONS}

The wire crystals were grown on n-type $\mathrm{GaAs}(111) \mathrm{B}$ substrates using $\mathrm{AsH}_{3}$ and TMG. The substrates were doped with $1 \times 10^{18} \mathrm{~cm}^{-3}$ of $\mathrm{Si}$. The growth occurred along the [111] direction such that the wire crystal axes were exactly perpendicular to the substrates. The

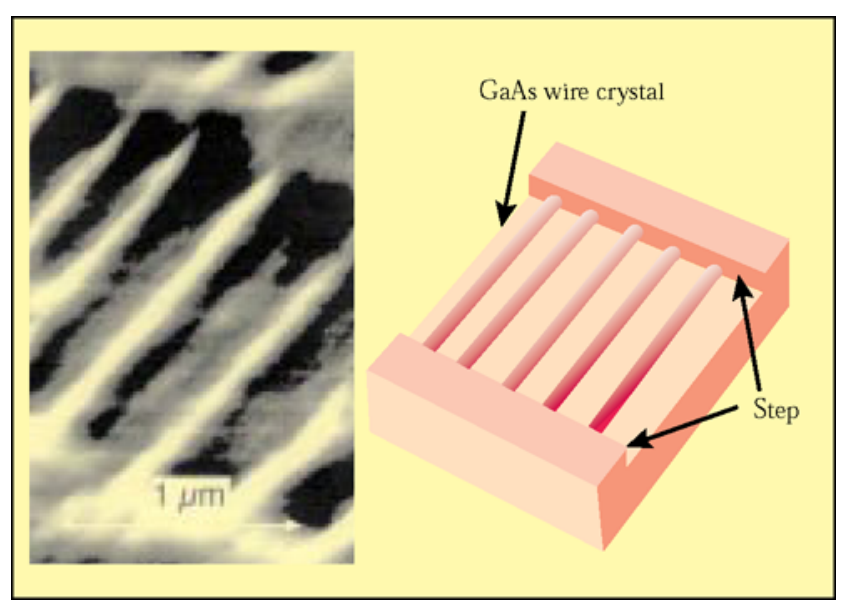

Figure 6 Bridge-type GaAs whisker arrays. 


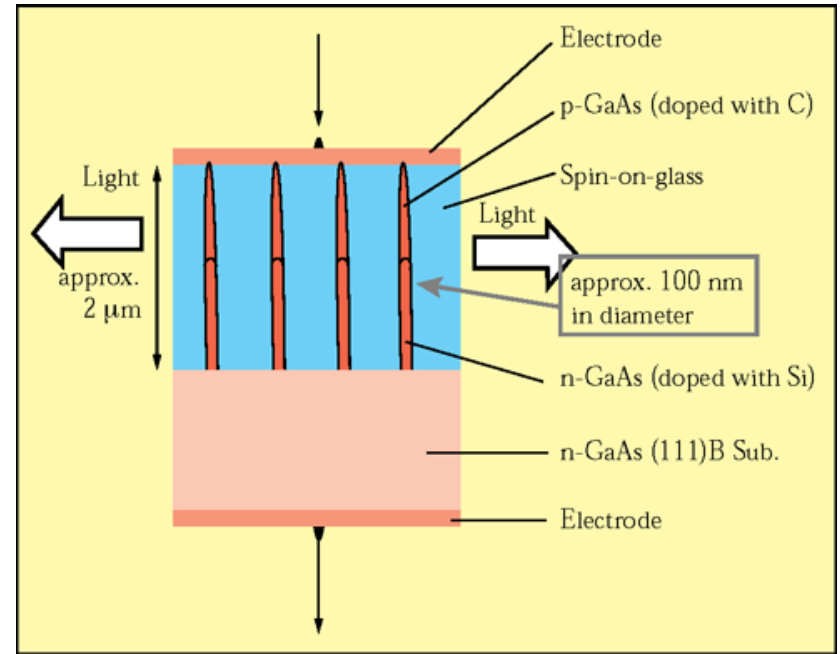

Figure 7 Fabrication of an LED with a whisker-diode structure.

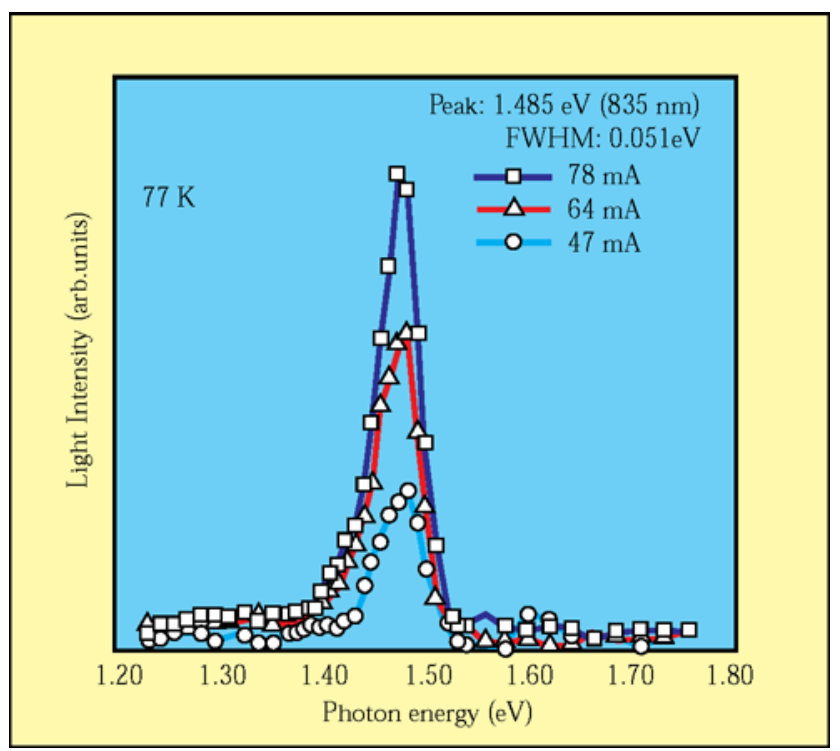

Figure 8 Emission spectra, measured at $77 K$ while current was applied.

length of the wire crystals was ca $2 \mu \mathrm{m}$ and their diameter ca $100 \mathrm{~nm}$. During the growth process, doping species were changed in order to obtain the p-n junction at the middle point of the wire crystal (13). During the first half of the wire growth, silicon was doped into the wire by using disilane $\left(\mathrm{Si}_{2} \mathrm{H}_{6}\right)$. This resulted in n-type doping of about $1 \times 10^{18} \mathrm{~cm}^{-3}$. Carbon doping also occurred because of the low supply rate of $\mathrm{AsH}_{3} / \mathrm{TMG}$ during the latter half of growth, thus leading to p-type doping of about $8 \times 10^{18} \mathrm{~cm}^{-3}$. Carbon doping is advantageous because it prevents the self diffusion of the dopant and reduces the diffusion of $\mathrm{Zn}$ from the $\mathrm{Au} / \mathrm{Zn}$ electrode used for the ohmic contact.

Figure 7 illustrates schematically a fabricated LED with a whisker-diode structure. There were $3.3 \times 10^{6}$ crystals per $1 \mathrm{~mm}^{2}$ area of this device. A conventional $\mathrm{Au} / \mathrm{Ge} / \mathrm{Ni}$ electrode was employed to form an ohmic contact on the substrate side. $\mathrm{Au} / \mathrm{Zn}$ electrodes were fabricated as ohmic contacts on the top of the p-type doped wire crystal. The wire crystals were buried under spin-on-glass (SOG).

We measured electrical characteristics of the device. A sudden rise in current was observed at $0.6 \mathrm{~V}$ forward bias. Moreover, soft breakdown occurred at $c a$ $-2.5-\mathrm{V}$ reverse bias. The device thus showed the nonlinear I-V characteristics inherent in a p-n junction.

Such a $p-n$ junction can be characterized by electroluminescence measurements (13). Emission spectra were measured at $77 \mathrm{~K}$ while current was applied (Figure 8). It can be seen from the figure that emission intensity varies linearly with injection current density. The peak position for all three currents is at $1.485 \mathrm{eV}(835 \mathrm{~nm})$. The width at half maximum remained constant at $0.051 \mathrm{eV}$, even with varying current. The peak energy of light emitted from bulk GaAs with carbon doping is estimated to appear at $1.483 \mathrm{eV}(836 \mathrm{~nm})$. This coincidence explains why the emitted light observed here comes from the $p-n$ junction. Evidence of the quantum confinement effect is the polarization dependence of the electroluminescence. The electroluminescence spectra

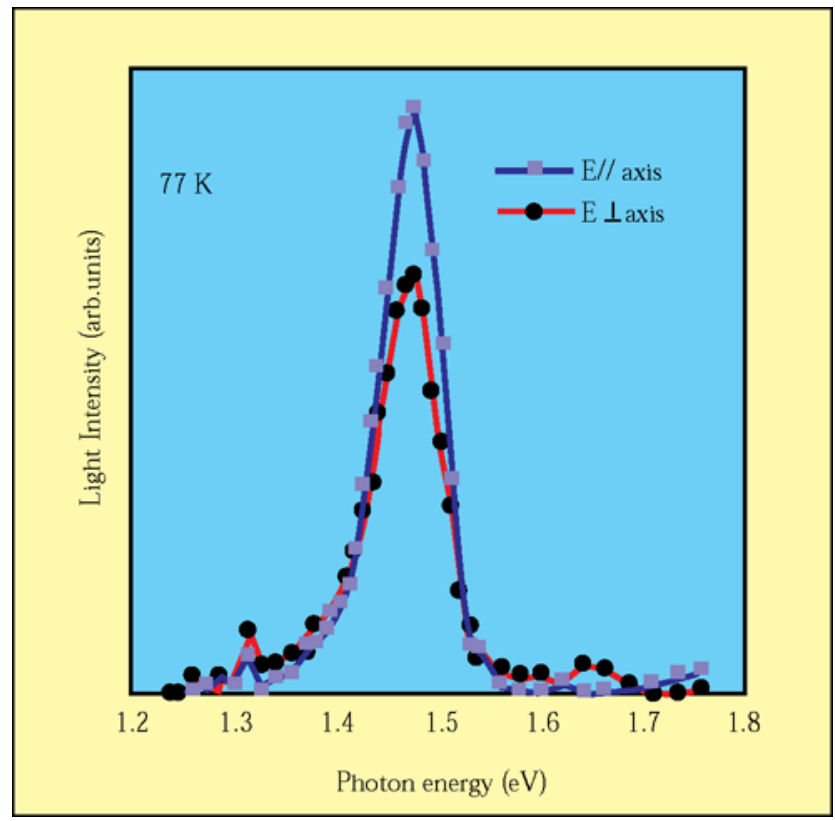

Figure 9 Electroluminescence spectra at $77 \mathrm{~K}$. 
at $77 \mathrm{~K}$ are given in Figure 9 (14). The solid line represents the electroluminescence spectrum for the light whose electric field vector is parallel to the wire crystal axis, while the dashed line indicates the spectrum of the electric field vector perpendicular to the wire crystal. The peak intensity of perpendicular light is $30 \%$ smaller than that of parallel light at $77 \mathrm{~K}$. In the case of bulk samples with p-n junctions, we could not find any polarization anisotropy at this temperature $(77 \mathrm{~K})$. These results therefore suggest the existence of the two-dimensional quantum confinement of the carriers. However, it should be noted that the polarization anisotropy is much stronger than the measured ratio because of the waveguide structure of the sample having metal electrodes. The polarization anisotropy of each individual wire crystal was estimated to be $20 \%$ when sample geometry was considered. This value is in good agreement with that given by the cylindrical approximation theory proposed by Sercel et al. The anisotropy is clear evidence of the quantum confinement effect of the carriers.

\section{CONCLUSIONS}

GaAs wire crystals as thin as $15-50 \mathrm{~nm}$ have been grown by metal-organic vapour-phase epitaxy. The growth mechanism is based on the vapour-liquid-solid (VLS) growth model. Gold alloy droplets play an essential role in the crystal growth. The crystal growth site on the substrate can be artificially controlled by positioning the gold alloy droplets. Nanolithography techniques have been used to laterally grow these wire crystals. Furthermore, GaAs whiskers with p-n junctions were grown. These $p-n$ junctions emit intense light, which shows that $\mathrm{GaAs}$ whiskers can be used as ultra-small light-emitting diodes.

\section{ACKNOWLEDGEMENTS}

This work was performed under the management of FED as a part of the MITI R\&D Program (Quantum Functional Devices Project) supported by NEDO.

\section{ABOUT THE AUTHORS}

Masataka Shirai received his MS degree from the University of Tokyo in 1993. Since then he has been working at the Central Research Laboratory of Hitachi Ltd. His current activity is in the development of optoelectronic devices for fibre optic communications.

Kei-ichi Haraguchi received his BS degree in electronics from Waseda University, Tokyo in 1991. He has been working at Hitachi on silicon VLSI devices.

Kenji Haruma received his $\mathrm{PhD}$ degree in Applied Physics from the University of Tokyo in 1982. He has been at Hitachi since April 1999 working on crystal growth technology for $\mathrm{GaAs}$ and its related compound semiconductors.

Toshio Katsuyama obtained his $\mathrm{PhD}$ in Applied Physics from Tohoku University, Sendai, Japan in 1983. Since 1974 he has been working for Hitachi, engaged in research on optical fibres, semiconductor integrated optics and quantum functional devices.

\section{REFERENCES}

1 M. Tanaka and H. Sasaki, Appl. Phys. Lett.,1989, 54, 1326

2 N. Randoll, M.A. Reed and Y.C. Kao, J. Vac. Sci. Technol, 1990, B8, 1348

3 Y. Hirayama, Y. Suzuki, S. Tarucha and H. Okamoto, Jpn. J. Appl. Phys., 1985, 24, L.516

4 A. Scherer, M.L. Roikes, H.G. Graighead, R.M. Ruthen, E.D. Beebe and J.P. Habison, Appl. Phys. Lett., 1987, 51, 2133

5 H. Asad, S. Yamada and T. Fukui, Appl. Phys. Lett., 1987, 51, 1518

6 M. Yazawa, M. Koguchi and K. Hiruma, Appl. Phys. Lett, 1991, 58, 1080

7 K Hiruma, T. Katsuyama, K. Ogawa, M. Koguchi, H. Kakibayashi and G.P. Morgan, Appl. Phys. Lett, 1991, 59, 431

8 R.S. Wagner and W.C. Ellis, Appl Phys. Lett, 1965, 4, 89

9 E.I. Givargizov, J. C.rgstal Growth, 1975, 31, 20

10 T. Sato, K. Hiruma, M. Shira, K. Tominaga, K Haraguchi, T. Katsuyama and T. Shimada, Appl. Phys. Lett, 1995, 66, 159

11 K. Haraguchi, K. Hiruma, T. Katsuyama, K. Tominaga, M. Shirai and T. Shimada, Appl. Phys. Lett, 1996, 69, 386

12 K. Haraguchi, T. Katsuyama, K. Hiruma and K. Ogawa, Appl. Phys. Lett, 1992, 60, 745

13 K. Haraguchi, T. Katsuyarna and K. Hiruma, J. Appl Phys, 1994, 75, 4220

14 T. Katsuyama, K. Hiruma, T. Sato, K. Ogawa, M. Shira, K. Haraguchi and M. Yazawa, Topical Meeting of Quantum Optoelectronics, Dana Point, California, 1995 